\title{
Logics and their galaxies
}

\author{
Hilan Bensusan, Alexandre Costa-Leite and Edelcio G. de Souza
}

\begin{abstract}
This article introduces some concepts that help exploring the ontological import of universal logic. It studies the notions of an antilogic and counterlogic associated to each logic and shows some of their properties. It presents the notion of galaxy, as the class of possible worlds compatible with a given logic. We explore some consequences of these developments.
\end{abstract}

Mathematics Subject Classification (2000). Primary 03B22; Secondary 03A99.

Keywords. Universal Logic, Antilogics, Counterlogics, Galaxies.

\section{Towards a universal metaphysics}

Metaphysicians make constant use of logic. They need it to make claims about what is $\log$ ically possible, what is logically necessary or contingent. They need it to reason on what ought to be the case once some assumptions are made - issues of logical compossibility. They need it whenever they investigate a priori knowledge and whenever they appeal to sufficient reason. Without an underlying logic no thesis concerning contingency, compossibility, necessity or sufficient reason can be substantial. The use of logic by metaphysics is evident when we consider the framework of possible worlds - taken by David Lewis [11], pp. 1-4, to be a paradise for philosophers. At least since Lewis work, back in the 70s, metaphysicians have been making use of possible worlds to discuss and evaluate claims and arguments concerning several topics such as causation ([10], [9]), dispositions ([13]), monism and pluralism ([4]), semantics ([16]) or the existence of necessary objects ([22]). The appeal to possible worlds, however, hinges always on a distinction between possible and impossible worlds (distinction drawn by Lewis himself [11], p. 1). A logically impossible world can arguably be dismissed from metaphysical considerations.

Now, there are different logics. In fact, an infinite number of them. Clearly, as metaphysics is entangled with logic, different underlying logics produce different results in metaphysics. Things are evaluated differently and distinct claims are made possible when we move from one logic to another. To see this, it is enough to focus on what makes a

Work supported by a grant of the National Council for Scientific and Technological Development - CNPq Brazil (486635/2013-9). 
world impossible. What is impossible in classical logic is not necessarily so in paraconsistent or intuitionistic logics. The question arises then as to which logic to choose. In general, metaphysicians have dealt with this in an easy and oversimplified way: just use classical logic. To be sure, nevertheless, the choice of a logic is a tricky issue that, at the face of it, involves circularity: we cannot argue that one particular logic is somehow necessary without appeal to an underlying logic. Given these difficulties, it is quite common to dismiss the question altogether by saying that in the absence of any reason to select any other logic, classical logic is best retained. Such a response, nevertheless, has been challenged by recent developments in universal logic.

Universal logic looks at the plurality of logics not in order to choose one among them but rather to study the relations between them. It explores how to compare them, how to put them together and how to build new logics out of known ones. It aims at a general investigation of logics in a way that is similar to what has been achieved with universal algebra (see [1]). To this purpose, universal logic has been using methods such as the combination of logics, techniques of translating logics and tools in category theory. It looks at the space of all logics and how it constrains the scope of a particular logic.

The present article begins to explore the idea that universal logic - and not any logic in particular - should underlie the efforts of metaphysicians. It points towards a metaphysics grounded on universal logic - a universal metaphysics. We begin our exploration by considering possible worlds in a framework where different logics take different worlds to be impossible. In order to do that, we develop the notion of a galaxy compatible with a given logic - a galaxy is a class of possible worlds. It is a way to formalize the notion of underlying logic of a theory. If we are right, metaphysicians should be looking at galaxies and their properties whenever they are making claims involving possibility, necessity and related concepts.

\section{Logics, antilogics and counterlogics}

In order to consider the infinite number of logics and to make evident how different logics yield different ontological commitments, we make use of the notions of antilogic and counterlogic defined for a suitable logic. Here we will not consider any logic in particular, but rather a logic in an abstract sense.

A logic $L$ is a structure $\left(F, \vdash_{L}\right)$ such that $F$ is a set and $\vdash_{L}$ is a binary relation on $\wp(F) \times F$ without any restriction. ${ }^{1}$ We use $\Gamma \vdash_{L} \varphi$ to indicate that $(\Gamma, \varphi) \in \vdash_{L}$ and we say that $\varphi$ is a consequence of $\Gamma$ in $L$. So, $\vdash_{L}$ is called a consequence relation of $L$.

The antilogic of a given logic $L$ is denoted by $\bar{L}^{2}$ The counterlogic of a given logic $L$ is denoted by $\tilde{L}$. Antilogics and counterlogics are also logics in the sense that they are sets and consequence relations without axioms. If we define logics using consequence relations with Tarskian conditions, then antilogics and counterlogics would not be logics.

\footnotetext{
${ }^{1}$ Tarski proposed an operator of logical consequence with conditions on it (see [20]). We use a consequence relation without restrictions in the sense of [2].

${ }^{2}$ The concept of antilogic has been developed by Łukasiewicz in [12], and studied in [17] as rejected propositions or in [18] under the label of refutation systems. For anticlassical propositional logic, there are results in [6], [21] and [3].
} 
The antilogic $\bar{L}$ of a given logic $L=\left(F, \vdash_{L}\right)$ is a pair $\left(F, \vdash_{\bar{L}}\right)$ such that

$\Gamma \vdash_{\bar{L}} \varphi$ if and only if it is not the case that $\Gamma \vdash_{L} \varphi$.

It is clear that for each $L$ there is exactly one $\bar{L}$. Moreover considering that consequence relations are sets of pairs, a natural consequence of the above definitions is that there is nothing in common between the set corresponding to a logic and the one corresponding to its antilogic:

$$
\begin{aligned}
\vdash_{L} \cap \vdash_{\bar{L}} & =\varnothing ; \\
\vdash_{L} \cup \vdash_{\bar{L}} & =\wp(F) \times F .
\end{aligned}
$$

This means that the two sets cover all the well-formed formulas expressed in the vocabulary where $L$ (and of course, $\bar{L}$ ) is defined. In this sense (but not necessarily in another to be considered in the next section involving the concept of galaxies), we can say that $\bar{L}$ is the complement of $L$.

Let $L=\left(F, \vdash_{L}\right)$ be a logic such that $F$ is closed by an unary operation of negation, that is, if $\varphi \in F$, then $\neg \varphi \in F$. In this case, we say that $L$ is a logic with negation. Given a logic with negation $L=\left(F, \vdash_{L}\right)$, the $\neg$-counterlogic ${ }^{3}$ of $L$ is the logic given by the pair $\tilde{L}=\left(F, \vdash_{\tilde{L}}\right)$ such that:

$$
\Gamma \vdash_{\tilde{L}} \varphi \text { if and only if } \Gamma \vdash_{L} \neg \varphi
$$
one $\tilde{L}$.

Again, it is clear that for each $L$, and for each negation operation, there is exactly

We already have elements to prove that soundness and completeness are preserved from a logic to its antilogic and its counterlogic.

Theorem 2.1. If $L=\left(F, \vdash_{L}\right)$ is sound and complete with respect to a semantic structure (which is also a logic in the sense above) $S=\left(F, \vDash_{S}\right)$, that is, if it holds that: $\Gamma \vdash_{L} \varphi$ if and only if $\Gamma \vDash_{S} \varphi$; then, $\bar{L}$ is sound and complete with respect to $S=\left(F, \vDash_{\bar{S}}\right)$, such that $\vDash_{\bar{S}}$ is given by $\Gamma F_{S} \varphi$ if and only if it is not the case that $\Gamma F_{\bar{S}} \varphi$. A similar result can be stated to counterlogics.

As for interactions of a logic with negation, its antilogic and its counterlogic, it is important to note that:

$$
\begin{aligned}
& \overline{\bar{L}}=L ; \\
& \tilde{\tilde{L}}=L ; \\
& \overline{\tilde{L}}=\tilde{\tilde{L}} ;
\end{aligned}
$$

$\Gamma \vdash_{\bar{L}} \varphi$ if and only if it is not the case that $\Gamma \vdash_{\tilde{L}} \neg \varphi$;

$\Gamma \vdash_{\tilde{L}} \varphi$ if and only if it is not the case that $\Gamma \vdash_{\bar{L}} \varphi$.

Notice also that, as the antilogic and the counterlogic are defined in terms of the consequence relation, a logic that entails nothing $\left(\vdash_{L}=\varnothing\right)$ is its own counterlogic. Moreover, its antilogic is the trivial logic $\left(\vdash_{L}=\wp(F) \times F\right)$.

\footnotetext{
${ }^{3}$ Henceforth, whenever non-ambiguous we use simply "counterlogic".
} 


\section{Antilogic, counterlogic and the square of oppositions}

The antilogic and the counterlogic of a logic can define interesting oppositions concerning relations between logics. These oppositions can be thought in terms of the framework of the square of oppositions. Such a framework (and its extensions in the so-called geometry of oppositions (see [14]) has been used to study all sorts of relations of opposition. Different logics are such that they can hold relations analogous to those of contradiction, contrariety and subalternity between them. In this section, we begin to study these relations of oppositions by seeing different logical systems as poles in a triangle or a square. By presenting a square of logics that capture some of their relations, we pave the way for a more systematic study of the oppositions between logics.

We start with some brief remarks concerning the square. We call a square complete if it is a square with all four oppositions: contradiction, contrariety, sub-contrariety and subalternation. A square is standard if it fits any family of concepts satisfying traditional oppositions. A square is perfect if it is complete and standard. Moreover, any square which is not complete or/and standard is called degenerate square.

We can now explore the oppositions between a logic and its counter and antilogic in terms of the square. The main question is to determine what are the oppositions between these logics.

Because the vertices of the square (or of the triangle formed by a logic, its counterlogic and its antilogic) are not propositions we reconstruct the classical oppositions accordingly. We define them in terms of relations between logics - instead of truth-values.

First, let $L_{1}=\left(F, \vdash_{L_{1}}\right)$ and $L_{2}=\left(F, \vdash_{L_{2}}\right)$ be two logics. We say that $L_{1}$ is a sublogic of $L_{2}$ if and only if $\vdash_{L_{1}} \subseteq \vdash_{L_{2}}$. We use the following notation: if $\vdash_{L} \subseteq \wp(F) \times F$, then $\nvdash_{L}$ is the complement of $\vdash_{L}$ with respect to $\wp(F) \times F$.

Now we introduce some relations between logics:

(d) $L_{1}$ and $L_{2}$ are contradictories if and only if $\vdash_{L_{1}} \cap \vdash_{L_{2}}=\varnothing$ and $\nvdash_{L_{1}} \cap \nvdash_{L_{2}}=\varnothing$;

(c) $L_{1}$ and $L_{2}$ are contraries if and only if $\vdash_{L_{1}} \cap \vdash_{L_{2}}=\varnothing$ and $\nvdash_{L_{1}} \cap \nvdash_{L_{2}} \neq \varnothing$;

(sc) $L_{1}$ and $L_{2}$ are subcontraries if and only if $\vdash_{L_{1}} \cap \vdash_{L_{2}} \neq \varnothing$ and $\nvdash_{L_{1}} \cap \nvdash_{L_{2}}=\varnothing$;

(s) $L_{1}$ is subaltern to $L_{2}$ if and only if $L_{2}$ is a sublogic of $L_{1}$.

It is not straightfoward to present oppositional structures for any logic. We will proceed by introducing some restrictions. First, we restrict ourselves to logics which accept elimination of double negation in an obvious sense. Additionally, let $L$ be a logic with negation. We say that $L$ is well-behaved if and only if for every pair $(\Gamma, \varphi)$, it is not the case that $\left(\Gamma \vdash_{L} \varphi\right.$ and $\left.\Gamma \vdash_{L} \neg \varphi\right)$.

Theorem 3.1. If $L$ is well-behaved then $\tilde{L}$ is a sublogic of $\bar{L}$.

For, suppose that $\Gamma \vdash_{\tilde{L}} \varphi$. By definition, $\Gamma \vdash_{L} \neg \varphi$. Since $L$ is well-behaved, this is not the case that $\Gamma \vdash_{L} \varphi$. Therefore, $\Gamma \vdash_{\bar{L}} \varphi$. 
Let $L=\left(F, \vdash_{L}\right)$ be a logic with a negation. Consider $\varphi \in F$. We say that $L$ is $\varphi$-silent if and only if we have neither $\varnothing \vdash_{L} \varphi$ nor $\varnothing \nvdash_{L} \varphi$. $L$ is called silent if and only if there exists $\varphi \in F$ such that $L$ is $\varphi$-silent. Notice that if $L$ is silent then its corresponding $\bar{L}$ is not well-behaved.

We can start out by remarking that $L$ and $\bar{L}$ are contradictories. Also, if $L$ is wellbehaved and with double negation elimination rule, $L$ and $\tilde{L}$ are contraries. Notice that $\tilde{L}$ is then also well-behaved. Since $L$ is well-behaved, $\tilde{L}$ is a sublogic of $\bar{L}$ and therefore the former is subaltern to the latter. We can then have a triangle of oppositions for a wellbehaved $L$ :

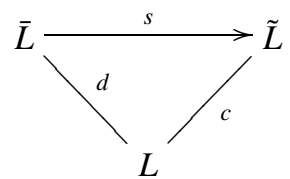

We can now consider a fourth vertice. Consider $\overline{\tilde{L}}$ which we have seen is equivalent to $\tilde{\bar{L}}$. Clearly, for any $L$ that is well-behaved, silent and with double negation elimination rule, so is $\tilde{L}$. Then we can see that if $L$ is well-behaved, $\tilde{\tilde{L}}$ is a sublogic of $\tilde{\tilde{L}}$, by the theorem above. As $\tilde{\tilde{L}}=L$, then $L$ is subaltern to $\overline{\tilde{L}}$ (and therefore to $\tilde{\tilde{L}}$ ). Further, if $L_{1}$ and $L_{2}$ are contraries, then $\bar{L}_{1}$ and $\bar{L}_{2}$ are subcontraries.

We can then present a square of oppositions for a well-behaved, silent with double negation $L$ :

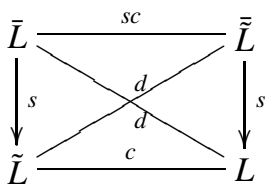

This square of logics displays oppositions between any well-behaved, silent and accepting double negation logic on the one hand and three other logics constructed from it on the other. It is a complete square, but not a standard one and therefore it is a degenerate square. To be sure, it could be possible to find a perfect square of logics by extending differently the triangle above. However, the degenerate square is enough to show how any given well-behaved and silent logic is accompanied by four other ones in relations of opposition. It is a square of logics showing how different (and yet related) logics are in relations of opposition.

\section{Galaxies}

The relations between any logic and its corresponding antilogic and counterlogic provides a convenient set-up to investigate the nature of logical necessity and therefore what 
counts as a possible world outside classical logic. It is clear that to each logic there is an associated class of possible worlds. To think of different logics in terms of possible worlds enable us to consider not only (accessibility) relations between different worlds made possible by a single logic but also relations between worlds made possible by different logics. The connection between a logic and its associated class of possible worlds began to be made explicit (and fruitful) by Kripke's semantics.

Indeed, in modal logic, it is customary to understand a logic in terms of the possible worlds associated to it (see [5]). In Kripke's semantics, different modal logics (primarily the normal ones $\mathbf{K}, \mathbf{T}, \mathbf{S 4}$ and $\mathbf{S 5}$ ) are each associated to the different accessibility relations between worlds and therefore to a class of possible worlds. What discriminates between those classes is the type of accessibility relations between worlds that they feature. In general, modal logics makes it clear that classes of possible worlds are associated to consequence relations. The more abstract idea is that a consequence relation can be understood in terms of an associated class of possible worlds. To these classes we turn now.

Let $\mathcal{L}$ be a class of all logics. Consider, also, a class $\mathcal{W}$ which elements will be called possible worlds. Let $\Vdash$ be a binary relation in $\mathcal{W} \times \mathcal{L}$. We call this compatibility relation. When $w \Vdash L$ we say that the possible world $w$ is compatible with the logic $L$.

Let $G$ be a function (of classes) defined as:

$$
\begin{aligned}
G: \mathcal{L} & \rightarrow \wp(\mathcal{W}), \\
L & \mapsto G(L):=\{w \in \mathcal{W}: w \Vdash L\} .
\end{aligned}
$$

Therefore, given a $\operatorname{logic} L, G(L)$ is the set of possible worlds compatible with $L$. $G(L)$ is called the galaxy of $L$. Notice that for each logic $L$ associated to a single consequence relation there is one and only one $G(L)$. A galaxy can be seen as the ontological counterpart of a logic.

The following condition on the compatibility relation has to be satisfied:

(SC) Sublogic Condition: Consider that $L_{1}$ is a sublogic of $L_{2}$, if $w \Vdash L_{1}$, then $w \Vdash L_{2}$.

Further, we can also introduce an extra condition relating a logic and its antilogic:

(SCC) Strong Constraint Condition: For every $w \in \mathcal{W}, w \Vdash L$ if and only if it is not the case that $w \Vdash \bar{L}$.

Under (SCC), we have:

$$
\begin{gathered}
G(L) \cap G(\bar{L})=\varnothing ; \\
G(L) \cup G(\bar{L})=\mathcal{W} .
\end{gathered}
$$

(SCC), though, could be too strong for it fails to capture the standard way to talk about possible worlds. To see this, consider the following toy example. Let $L=\left(F, \vdash_{L}\right)$ be a logic such that $F=\{\alpha, \beta\}$. Suppose further that $\varnothing \vdash_{L} \alpha$ and it is not the case that $\varnothing \vdash_{L} \beta$. Therefore, we have $\varnothing \vdash_{\bar{L}} \beta$ and it is not the case that $\varnothing \vdash_{\bar{L}} \alpha$. Let $w_{1} \in \mathcal{W}$ and $w_{2} \in \mathcal{W}$ 
be possible worlds such that $\alpha$ and $\beta$ are the case in $w_{1}$, and $\alpha$ and $\beta$ are not the case in $w_{2}$. It would be reasonable to consider that for all $w \in \mathcal{W}, w \in G(L)$ if and only if for any $\varphi \in F, \varnothing \vdash_{L} \varphi$ implies that $\varphi$ is the case in $w$. Thus, in our toy example, $w_{1} \in G(L)$ and $w_{2} \notin G(L)$ while $w_{1} \in G(\bar{L})$ and $w_{2} \notin G(\bar{L})$. In other words, $G(L) \cap G(\bar{L}) \neq \varnothing$, and $G(L) \cup G(\bar{L}) \neq \mathcal{W}$.

It can be therefore natural to weaken the constraint on the compatibility relation. An alternative condition would then be

(WCC) Weak Constraint Condition: Given a logic $L$ and its antilogic $\bar{L}$, for every $w \in W$, it is not the case that $(w \Vdash L$ if and only if $w \Vdash \bar{L})$.

It is interesting to consider how the galaxy of a logic and of its counterlogic relate. It could be that

$$
G(L) \cup G(\tilde{L}) \neq \mathcal{W} .
$$

Neither a logic and its respective counterlogic can cover all formulas where the logic is defined nor their respective galaxies can cover all worlds. Also, it could be that

$$
G(L) \cap G(\tilde{L}) \neq \varnothing .
$$

When this is the case, this is a remarkable intersection class for it is composed of only inconsistent worlds. All logics that admit inconsistencies are such that their galaxies are in this intersection.

So, let a class of all inconsistent logics be defined as such:

$$
\Delta=\left\{L \in \mathcal{L}: \text { there is a } \varphi \text { such that } \varnothing \vdash_{L} \varphi \text { and } \varnothing \vdash_{L} \neg \varphi\right\} .
$$

If so we can say that for any $L$

$$
G(L) \cap G(\tilde{L}) \subseteq G(\Delta):=\bigcup\{G(L): L \in \Delta\} .
$$

In other words, the intersection between the galaxy of a logic and of its counterlogic is a subclass of the galaxy of all inconsistent logics. The intersection is always inconsistent. We call a world $w$ such that $w \in \Delta$ a dialethea, or an inconsistent world. ${ }^{4}$ It follows that

for each $w \in G(\Delta)$ there is an $L$ such that $w \in G(L) \cap G(\tilde{L})$.

These prospective remarks can be viewed as the starting point of an algebra of galaxies that is neither reducible nor isomorphic to the algebra of logics (or the algebra of consequence relations (see [19])). Such an algebra is a tool to study how different logics intersect with each other. Also, operations on galaxies reflect operations on logics - such as fusion (in the sense of combination of modal logics (see [8])). So we have:

$$
G\left(L_{1} \oplus L_{2}\right)=G\left(L_{1}\right) \cup G\left(L_{2}\right) .
$$

The algebra is a useful tool to look at the ontological issues raised by universal logic.

\footnotetext{
${ }^{4}$ The term is used here in a way that is reminiscent but not equal to that used by Priest (see [15]). He takes a dialethea to be a part of reality which harbours inconsistencies, and true contradictions. We take it to be simply an inconsistent world. It is also worth noticing that if $L$ is such that it is not the case that $\varnothing \vdash_{L} \varphi \wedge \neg \varphi$ then $\varnothing \vdash_{\bar{L}} \varphi \wedge \neg \varphi$. Therefore $\bar{L}$ could contain dialetheas (true contradictions) also in Priest's sense.
} 


\section{Prospects for a metaphysics of galaxies}

We can then say that a world is possible only with respect to a galaxy. This has an impact on the notion of logical truth - or necessity simpliciter as it is classically conceived given that (logical) truths are relative to galaxies. When we say that something is the case in all possible worlds we ought to specify a galaxy in which these possible worlds are. We can however say some general things through the framework of galaxies. A relevant general result is that for any $\varphi$, if $\varphi$ is contingent in a galaxy, there is a galaxy in which it is necessary.

A notion directly affected by understanding a possible world as relative to a galaxy is that of necessity. Either reduced to a single type (broadly constructed as metaphysical necessity or narrowly conceived as logical necessity) or as a multiplicity (where natural or normative necessity cannot be understood in terms of logical or metaphysical necessity ${ }^{5}$, necessity is understood in terms of classes of possible worlds. Now, it seems that it follows from consideration on galaxies that necessity is relative to a place in the space of galaxies. There is no such thing as absolute necessity. How would a metaphysics without a notion of absolute necessity look like?

This is the main metaphysical open problem raised by galaxy theory, i.e, the study of galaxies. It bifurcates into two different issues: the nature of modal claims under the framework of galaxies and the ontological status of galaxies. As for the first issue, modal claims in general appear to have to be indexed by galaxies. These claims become relative to a class of worlds. It is interesting to remark that the relative character of necessity entails also the relativity of contingency to galaxies. At first sight, a metaphysics that takes into consideration different galaxies of possible worlds is close to a Humean approach in that it exorcises necessity; and in fact it is more extreme than typical Humean approaches as it dismisses logical necessity as well. It would be a metaphysics of absolute contingency. However nothing happens contingently in all galaxies. There is no such thing as (logical) contingency simpliciter. If there is nothing but things that are contingent on something else, maybe other modal notions such as dependency and compossibility should rather be taken as basic. Those notions are relational ones and make explicit that modal ties are not absolute and have to be placed in the space of galaxies. Given a galaxy (or a collection of galaxies), we can study necessary (or accidental) connections within it. As for the overall picture where we ask questions about, say, the contingency of it all, they would have to be fully reformulated.

As for the second issue, we can make some sketchy remarks about the reality of galaxies. David Lewis has championed a version of modal realism ([11]) that maintains that all possible worlds exist - the actual one being no more than the one which happens to be this. There is no special property that renders any world actual, it is a world among others and it is actual for those who are in it. Actuality is indexical while existence is shared by all worlds (within a chosen galaxy - the classical one). From the point of view of galaxies, for a world to exist, it is enough to be in the right galaxy. Now, given the plurality of galaxies, one could formulate a stronger version of modal realism that would include

${ }^{5}$ Kit Fine ([7]) , for example, considers three irreducible varieties of necessity: metaphysical necessity, natural necessity and normative necessity. 
all worlds across all galaxies. One could be a realist concerning worlds possible in all logics and therefore extend realism to galaxies. If galaxies are part of the furniture of the universe, then the plurality of logics - the concern of universal logic - becomes a plurality not only about reality but in reality. In Lewis' realism about possible worlds, each world is actual for its citizens. Analogously, a galactic (modal) realism would take the actual world as no more than our current address. But then further questions concerning the plurality of logics in reality could be raised. The issue can be framed in terms of where does this world belong within the space of galaxies.

The plurality of galaxies is the opening door to a metaphysics informed by universal logic. This plurality doesn't entail the relativity of all metaphysical truths but rather guides the metaphysical gaze towards this plurality. Kit Fine ([7]) has studied three options with respect to the tension between a notion of reality that is neutral, absolute and coherent and the plurality of perspectives that arises from McTaggart's problems with the reality of tense. Those three alternatives could have counterparts in the context of the plurality of galaxies - and its connection to reality. The first would be to choose one among the many alternative galaxies and take it to be real. This would take reality not to be neutral and would embrace a galactical anti-realism constrasting with the modal realism formulated above. The second option would be to consider that each galaxy has its own reality - reality would then be no longer absolute but relative to each galaxy. The third option would be to consider a broader reality - Fine labels it über-reality - that encompasses all galaxies, even though that reality would by no means exhibit coherence. Fine himself favours the equivalent of this third option and explores some of its consequences. Here again, the third option is more clearly in line with what we mean by a universal metaphysics oriented by a galaxy theory. A metaphysics guided by universal logics attempts to investigate this über-reality formed by the multiple galaxies and the way they relate to each other.

The focus on galaxies enables us to consider metaphysical issues beyond a parochial point of view on necessity and contingency. It goes along with universal logic for it springs out from the idea that each logic has a class of possible worlds associated to it. Galaxy theory is the ontological counterpart of universal logic. The latter is the study of all logics while the former is the study of their respective galaxies. It is also the study of all logics through its associated class of possible worlds. Further, as we see it, it is not simply a subsidiary of the universal logic, but rather feeds its development and can guide its endeavours.

\section{References}

[1] BEZIAU, J.-Y. (1994). Universal logic. In Logica94 - Proceedings of the 8th International Symposium, T.Childers and O.Majer (eds), Prague, pp.73-93.

[2] BEZIAU, J.-Y. (2005). From consequence operator to universal logic: a survey of general abstract logic. In: Logica Universalis: Towards a general theory of logics, Birkhäuser.

[3] BEZIAU, J.-Y; BUCHSBAUM, A. (2013). Let us be Antilogical: Anti-Classical Logic as a Logic, in A.Moktefi, A.Moretti and F.Schang (eds), Let us be Logical, College Publication, London, 2013.

[4] BOHN, E. (2009). Must there be a top level? The Philosophical Quarterly, 59(235), 193-201. 
[5] BLACKBURN, P, M. DE RIJKE and Y. VENEMA. (2001). Modal Logic, Cambridge: Cambridge University Press.

[6] CAICEDO, X. (1978). A formal system for the non-theorems of the propositional calculus. Notre Dame Journal of Formal Logic (19), p. 147-151, 1978.

[7] FINE, K. (2005). Tense and reality. In Modality and Tense - Philosophical Papers, Oxford: Clarendon Press.

[8] GABBAY, D; KURUCZ, A. WOLTER, F. and ZAKHARYASCHEV, M. (2003). Manydimensional modal logics: theory and applications .Studies in Logic and the Foundations of Mathematics, Elsevier.

[9] HITCHCOCK, C. (2007). Prevention, preemption, and the Principle of Sufficient Reason. In: Philosophical Review, 116(4), 495-532.

[10] LEWIS, D. (1973). Causation. In Journal of Philosophy, 70, 556-67.

[11] LEWIS, D. (1986). On the Plurality of Words, Oxford: Blackwell.

[12] ŁUKASIEWICZ, J. (1951). Aristotle's Syllogistic from the Standpoint of Modern Formal Logic, Oxford: Clarendon Press.

[13] MARTIN, C. B. (1994). Dispositions and conditionals. In The Philosophical Quarterly, 44, $1-8$.

[14] MORETTI, Alessio. (2004). Geometry for modalities? Yes: through n-opposition theory. In: Aspects of Universal Logic, v. 17, p. 102-145.

[15] PRIEST, G. (2006). In Contradiction. Clarendon Press: Oxford.

[16] STALNAKER, R. (2012). Mere Possibilities. Princeton University Press.

[17] SŁUPECKI, J; BRYLL, G; WYBRANIEC-SKARDOWSKA, U. (1971). Theory of Rejected Propositions I. In Studia Logica, V. 29, pgs. 75-123.

[18] SKURA, T. (2009). A Refutation Theory. In Logica Universalis, vol. 3, pgs. 293-302.

[19] STONE, M. (1936). The theory of representation for boolean algebras. In: Transactions of the American Mathematical Society, vol. 40, p. 37-111.

[20] TARSKI, A. (2010). Remarks on Fundamental Concepts of the Methodology of Mathematics. In: Universal Logic: An Anthology, translated by R. Purdy and J. Zygmunt, Birkhäuser. Original: 1929.

[21] VARZI, A. (1992). Complementary logics for classical propositional languages. Kriterion (4),p. 20-24.

[22] WILLIAMSON, T. (2013). Modal Logics as Metaphysics, Oxford: Oxford UP.

\section{Acknowledgement}

Thanks to Arnold Koslow and Graham Priest for discussions concerning ideas of this paper. 
Hilan Bensusan

Universidade de Brasília

Campus Universitário Darcy Ribeiro

Instituto de Ciências Humanas

Departamento de Filosofia

ICC Ala Norte

Brasil

e-mail: hilanb@unb.br

Alexandre Costa-Leite

Universidade de Brasília

Campus Universitário Darcy Ribeiro

Instituto de Ciências Humanas

Departamento de Filosofia

ICC Ala Norte

Brasil

e-mail: costaleite@unb.br

Edelcio G. de Souza

Universidade de São Paulo

Departamento de Filosofia

Av. Prof. Luciano Gualberto, 315

Cidade Universitária - São Paulo - SP

Brasil

e-mail: edelcio.souza@usp.br 